\title{
Magnitude of Drug-Resistant Enterococcus Species from intestinal Tracts of Hospitalized Pediatric Patients in Debreberhan Referral Hospital, Debreberhan, Ethiopia
}

\author{
Sisay Gebrish ${ }^{1}$, BisratFikadu ${ }^{1}$, Tsegahun Asfaw ${ }^{2 *}$ \\ ${ }^{1}$ Department of Medical Laboratory Science, Debre Berhan Health Science College, Debre Berhan, Ethiopia \\ ${ }^{2}$ Department of Medical Laboratory Science, College of Medicine, Debre Berhan University, Debre Berhan, \\ Ethiopia
}

*Corresponding Author: Tsegahun Asfaw, Department of Medical Laboratory Science, College of Medicine, DebreBerhan University, DebreBerhan, Ethiopia.

\begin{abstract}
Background: Multi-drug-resistant enterococci are the major source of infection as well as nosocomial spread. There is scarcity of data ondrug-resistant enterococci in developing country including Ethiopia. Therefore, this study aimed to determine the magnitude of drug resistant enterococcus species from intestinal tracts of hospitalized pediatric patients.

Method: The study was conducted among hospitalized pediatric patients at DebreBerhanreferral hospital, from February 15 to March, 25 2016. Rectal swabs was collected and processed for bacterial isolation and susceptibility testing. The isolates was identified to species level by cultural characteristics, Gram's stain, catalase test and other biochemical tests. Susceptibility testing to antimicrobial agents was done using KirbyBauer disk diffusion method.

Result: Enterococci were isolated from $12(23 \%)$ of the study participants. The isolates were Enterococcus faecium(50\%),Enterococcus faecalis(33.3\%) and Enterococcus gallinarum(16.7\%). Among 12 tested Enterococci isolates, 5 (41.7\%) were resistant to ampicillin, 7(58.3\%) to streptomycin, 6 (50\%) to gentamycin. $7(58.3 \%)$ to ciprofloxacin, 5(41.7\%) to norfloxacin and 8(66.7\%) to erythromycin. Multiple drugresistance was observed among $75 \%$ of E. faeciumand E. faecalis. Vancomycin resistant Enterococci were observedin $16.7 \%$ of E. faeciumisolates.

Conclusion: This study reveals high rate of fecal colonization by multidrug-resistant enterococci and prevalence of vancomycin resistance strains. Thus periodic surveillance of antibiotic susceptibilities is recommended to detect emerging resistance and to prevent its spread.
\end{abstract}

Keywords: Magnitude, Drug-resistance, Enterococcus species, Pediatric Patients

\section{INTRODUCTIONS}

Enterococci are normal inhabitant of the gastrointestinal tract. However, they can also be significant pathogens causing several infections. The most common nosocomial infections caused by these organism are urinary tract infections (associated with instrumentations and antimicrobials administration) (1).The emergence of vancomycin resistance enterococci (VRE) is a global issue due to few option left for disease management. Besides drug resistant Enterococci can colonize the intestinal tract of hospitalized patients and become major source of infection as well as nosocomial spread $(2,3)$.

In humans, enterococcal infections may be caused by at least 12 species but most clinical infections are due to either Enterococcus faecalis or E. faecium. E. faecalisis the most common cause (80-90\%) followed by E. faecium(10-15\%). Occasional infections are due to Enterococcusgallinarum, Enterococcus raffinosus, Enterococcus casseliflavus, Enterococcus avium, Enterococcus pseudoavium, Enterococcus malodoratus, Enterococcus mundtii, Enterococcus durans, and Enterococcus hirae (4). The proportion of isolates of motile Enterococci (E.gallinarum, E. casseliflavus) is low. But they are intrinsically resistant to vancomycin and inappropriate treatment may contribute to morbidity and mortality (5). 
Several studies have documented that enterococcal infections are most commonly caused by the patient's own commensal flora. Colonization may occur long before or immediately before infection, but it plays a major role in the development of nosocomial infection (2).Despite the importance of these etiologic agents there is dearth of information regarding antimicrobial resistance of Enterococcus species isolated from intestinal tract of hospitalized patients in Ethiopia. Thus, the present study will be conducted to determine antimicrobial resistance pattern of fecal enterococci isolates from hospitalized pediatric patients.

\section{METHODS}

\subsection{Study Area and Periods}

The study was conducted at Debre Berhan referral hospital, from February 15, 2016 to march 25, 2016. It is located $130 \mathrm{kms}$ Northeast of Addis Ababa.

\subsection{Study Design}

Cross sectional study was conducted and the study participant was recruited conveniently.

\subsection{Sampling Technique and Sample Size}

52 patients, who had at least 2 days of hospital stay at pediatric ward of Debre Berhan referral hospital were enrolled by using convenience sampling technique.

\subsection{Inclusion and Exclusion Criteria}

Pediatric patients aged 0 to 15 years and having at least 48 hour hospital stay during the study period and fulfill the inclusion criteria was included while who cannot respond to the interview and for children without getting permission from guardian and consent was excluded.

\subsection{Data Collection Techniques}

Scio-demographic data was collected by pretested questionnaires after obtaining a written informed consent from study participants. Fecal samples were collected in sterile plastic stool containers. From critically ill patients rectal swabs were collected using sterile cotton swab moistened in sterile normal saline solution. Then, the swabs were immersed in well-labeled Cary- Blair semi-solid medium prepared in screw-capped tubes and transferred to the Debre Berhan university medical microbiology laboratory.

\subsubsection{Culture and Identification}

Stool specimens and rectal swabs were inoculated onto Bile Esculinazide agar plates with and without $6 \mu \mathrm{g} / \mathrm{ml}$ of vancomycin and incubated at $37^{\circ} \mathrm{C}$ for $24 \mathrm{~h}$. colonies with colourless or grey and surrounded by a black halo (hydrolysis of esculin) were sub-cultured and identified as Enterococci by additional tests like gram stain, catalase test, $6.5 \% \mathrm{NaCl}$ test, growth at $45^{\circ} \mathrm{C}$ and motility test as recommended by Facklam and Collins (6), Manero and Blanch (7). Identification of these isolates to species level was performed by API-20 Streptococcus system (bioMérieux).

\subsubsection{Antibiotic Susceptibility Testing}

Antimicrobial susceptibility studies were performed by disc diffusion (Kirby-Bauer) method according to Clinical Laboratory Standards Institute (CLSI) for widely used drugs in Ethiopia with their respective dose. Minimum inhibitory concentrations (MICs) for vancomycin were determined using E-test strips.

\subsection{Data Processing, Analysis}

The collected data was clearly summarized, filled and analysed by using SPSS version 21 . Descriptive statistics was employed to examine the finding, and the result was presented by using tables, charts and graphs. P-value less than or equal to 0.05 was considered as statistically significant value.

\subsection{Quality Assurance}

The questionnaires was pretested a week before actual data collection. The quality of reagents and equipment was checked and used according to manufacturer directions. The data was collected by trained data collectors and the result was recorded carefully and correctly. Standard operating procedure was applied during specimen collection, culture, drug susceptibility test and biochemical test. E. faecalis ATCC 29212 was used as a quality control strain for performing antimicrobial tests. 
Magnitude of Drug-Resistant Enterococcus Species from ntestinal Tracts of Hospitalized Pediatric Patients in Debreberhan Referral Hospital, Debreberhan, Ethiopia

\subsection{Ethical Consideration}

The study protocol was reviewed and approved by the ethical and review committee of Debre Berhan university. For all study participant the objective of the study was explained and written informed consent was obtained. Those positive for enterococcus was referred to their respective clinicians for further management.

\section{RESULT}

\subsection{Demographic Characteristics}

Among 52 participants, 27 (52\%) were males and 25(48\%) were females. The mean age of the patient's was 3 years. $78.8 \%$ study participant had a history of exposure to one or more antimicrobial agent in the last 2 weeks and $21.2 \%$ were without exposure and the average hospital stay was 17.5 days with a range of 2-45days (Table 1 ).

\subsection{Enterococci Isolates}

Among all participants, 12 (23\%) of the study participants were positive for at least one Enterococcus spp. There was no statistically significant association between isolation of Enterococci with age, sex, hospital duration and antibiotic history (Table 1).

Table1. Socio demographic characteristics and Enterococcus culture positivity at DebreBerhan Referral Hospital, DebreBerhan, Ethiopia

\begin{tabular}{|c|c|c|c|c|}
\hline Variable & CulturePositive n (\%) & CultureNegative $\mathrm{n}(\%)$ & Total $(\%)$ & $\mathrm{P}$-value \\
\hline \multicolumn{4}{|c|}{ Age category in year } & \\
\hline $0-7$ year & $8(25.8 \%)$ & $23(74.2 \%)$ & $31(59.6 \%)$ & \multirow[t]{2}{*}{0.432} \\
\hline $8-15$ year & $4(19 \%)$ & $17(81 \%)$ & $21(40.4 \%)$ & \\
\hline \multicolumn{4}{|l|}{ Sex } & \\
\hline Male & $7(26 \%)$ & $20(74 \%)$ & $27(52 \%)$ & \multirow[t]{2}{*}{0.546} \\
\hline Female & $5(20 \%)$ & $20(80 \%)$ & $25(48 \%)$ & \\
\hline \multicolumn{4}{|c|}{ Hospital duration } & \\
\hline $2-15$ day & $3(10.7 \%)$ & $25(89.3 \%)$ & $28(53.8 \%)$ & \multirow[t]{2}{*}{0.135} \\
\hline$>15$ day & $9(37.5 \%)$ & $15(62.5 \%)$ & $24(46.2 \%)$ & \\
\hline \multicolumn{4}{|c|}{ Previous antibiotic treatment } & \multirow{3}{*}{0.313} \\
\hline Yes & $7(17.1 \%)$ & $34(82.9 \%)$ & $41(78.8 \%)$ & \\
\hline No & $5(45.5 \%)$ & $6(54.5 \%)$ & $11(21.2 \%)$ & \\
\hline
\end{tabular}

\subsection{Species Distribution}

The distribution of species is that a total of 12 enterococcal isolates were obtained from 52 patients. The commonly enterococcal isolates were E. faecium(50\%) followed by E. faecalis(33.3\%) and E. gallinarum(16.7\%) (Table 2).

Table2. Distribution of Enterococcus species isolated from intestinal tract of hospitalized patients in Debre Berhan Referral Hospital, DebreBerhan, Ethiopia.

\begin{tabular}{|l|l|}
\hline Enterococcus species & $\mathbf{N}(\boldsymbol{\%})$ \\
\hline E. faecium & $6(50 \%)$ \\
\hline E. faecalis & $4(33.3 \%)$ \\
\hline E. gallinarum & $2(16.7 \%)$ \\
\hline Total & $\mathbf{1 2}$ \\
\hline
\end{tabular}

\subsection{Antimicrobial Resistance of Enterococcal Isolates}

\subsubsection{B-Lactam Resistance}

1/4 (25\%) E. faecalisand 4/6 (66.7\%) E. faecium were resistant to ampicillin. AllE. fecalisand5/6 (83.3) E. faeciumisolates were resistant to penicillin. 1/2 (50\%) occasional Enterococcus species (E. gallinarum) were resistant to penicillin.

\subsubsection{Aminoglycoside Resistance}

High-level resistance to gentamicin and streptomycin was detected by the high content disk. Gentamycin resistant were observed in $50 \%$ of E. faecalisand $66.7 \%$ of E. faecium. 2/4 (50\%) E. faecalisand $66.7 \%$ E. faeciumwere resistant to streptomycin. 
Magnitude of Drug-Resistant Enterococcus Species from ntestinal Tracts of Hospitalized Pediatric Patients in Debreberhan Referral Hospital, Debreberhan, Ethiopia

\subsubsection{Vancomycin Resistant Enterococci}

E. faecalisisolates was not resistance to vancomycin while $1(16.7 \%)$ of E. faecium isolates were resistant to vancomycin (Table-3).

Table 3. Antibiotic resistance profile of Enterococcus species at DebreBerhan Referral Hospital, DebreBerhan, Ethiopia

\begin{tabular}{|l|l|l|l|l|}
\hline \multirow{2}{*}{ Antibiotics } & \multicolumn{3}{l|}{ Resistant isolates (\%) } \\
\cline { 2 - 5 } & E. faecalis(n,4) & E. faecium $(n, 6)$ & Other species(n,2) & Total(n,12) \\
\hline Ampicillin $(10 \mu \mathrm{g})$ & $1(25 \%)$ & $4(66.7 \%)$ & $0(0 \%)$ & $5(41.7 \%)$ \\
\hline Penicillin $((10 \mu \mathrm{g})$ & $4(100 \%)$ & $5(83.3 \%)$ & $1(50 \%)$ & $10(83.3 \%)$ \\
\hline Gentamicin $((120 \mu \mathrm{g})$ & $2(50 \%)$ & $4(66.7 \%)$ & $0(0 \%)$ & $6(50 \%)$ \\
\hline Ciprofloxacin $(5 \mu \mathrm{g})$ & $3(75 \%)$ & $4(66.7 \%)$ & $0(0 \%)$ & $7(58.3 \%)$ \\
\hline Streptomycin $(300 \mu \mathrm{g})$ & $2(50 \%)$ & $4(66.7 \%)$ & $1(50 \%)$ & $7(58.3 \%)$ \\
\hline Erythromycin $((15 \mu \mathrm{g})$ & $1(25 \%)$ & $5(83.3 \%)$ & $2(100 \%)$ & $8(66.7 \%)$ \\
\hline Norfloxacin $(30 \mu \mathrm{g})$ & $1(25 \%)$ & $3(50 \%)$ & $1(50 \%)$ & $5(41.7 \%)$ \\
\hline Vancomycin $(6 \mu \mathrm{g} / \mathrm{ml})$ & $0(0 \%)$ & $1(16.7 \%)$ & $0(0 \%)$ & $1(8.3 \%)$ \\
\hline
\end{tabular}

\section{DISCUSSIONS}

This study investigated the prevalence and antibacterial resistance patterns of enterococci isolated from fecal samples of hospitalized pediatrics patients. In this study the distribution of enterococcus isolates were E. faecium (50\%),E. faecalis $(33.3 \%)$ and E. gallinarum(16.7\%). This is comparable to the study done in Brazil (25). But disagreement with reports from United States (8).In this study, the predominant enterococcus isolates was E. faecium. Study in Singapore has also reported an increase in E. faeciumfrom 78.9 to $91.8 \%$ over a period of 5 years from 2006 to 2010 from clinical cultures (9). Another study from India has also reported $66 \%$ E. faeciumfrom blood sample (10). In this study the prevalence of E. gallinarumwas $16.7 \%$ which is higher than study done in Ethiopia (1). This might be indicate that, even though enterococci including E. gallinarumsare infrequently isolated from clinical specimens, they have been implicated in a wide variety of invasive infections in humans, especially immune compromised or chronically ill patients.

In this study, E.faecalisisolates showed $25 \%$ resistance rate to ampicillin. It is higher than the resistance rates reported in Kuwait, Hong Kong and Brazil, which is $0-8.3 \%(11,12,13)$, and lower than $60.7 \%$ reported from Gaza (14). Resistance rates to ampicillin was observed in $66.7 \%$ of E. faeciumisolates which is comparable with study done in Gaza $66.7 \%$ (14). However, lower than study reported from Israel (15). All E.facalis and 83.3\% E. faeciumisolates were resistant to penicillin which is similar to study done in India from clinical isolates (10). The reason for higher prevalence of $\beta$ lactam antibiotic resistance in this study might be due to chronic cases and wider usage of broad spectrum antibiotics relative or enterococcus isolates possess an intrinsically relative resistance to penicillin and ampicillin. Furthermore, E. faeciumis less susceptible to $\beta$-lactam agents than E. faecalisbecause their penicillin-binding proteins (PBPs) have lower affinities for these antibiotics and some strains have plasmid-encoded $\beta$-lactamase.

Aminoglycosides are frequently used in combination with cell wall active antibiotics for severe enterococcal infections. Since enterococcal resistance to gentamicin and streptomycin occurs by different mechanisms, it is important to test susceptibility to both agents. Enterococci with high level resistance to streptomycin are susceptible to gentamicin. And also gentamicin resistance is a good predictor of resistance to other aminoglycosides except streptomycin (16). In this study, E. faecalisand E. faecium showed resistance for many drugs. Concomitant resistance of high level aminoglycoside resistance (HLAR) strains to the $\beta$-lactam antibiotic (ampicillin) was quite higher $(25 \%$ of $\mathrm{E}$. faecalisand $66.7 \%$ of E. faeciumstrains). This finding is a cause of concern, because the synergistic activity of the combination of $\beta$-lactam antibiotics with HLAR in the treatment of enterococcal infections is totally abolished. In such instances, controlling the spread of these organisms have supreme importance.

In this study $58.3 \%$ of enterococci isolate were resistant to ciprofloxacin and $41.7 \%$ of the isolates were resistant to norfloxacin. Other alternative antibiotics to treat infection by enterococcus also showed high rates of resistance (erythromycin (66.7\% of resistance) and $(58.3 \%)$ of resistance to streptomycin). The high rates of resistance in present study might be due to excessive or in appropriate use of those antibiotics for empirical treatment of mixed nosocomial infections caused by enterococci. 
The emergence of VRE is also due to the inappropriate use of cephalosporin as well as poor hospital infection control measures .This study showed $16.7 \% \mathrm{E}$. faeciumresistantto vancomycin which is higher than study done in Egypt 4\% (17), Iran 6.2\% (18), South Africa 10.2\% (19) and report from Korea 12\% (20) and lower than report from Turkey $34.8 \%$ (21). The possible reason for the emergence of VRE in this study might be due to antibiotic selective pressure because the patients had long duration in hospital and high rate of antibiotics treatment.

The increase of invasive infections caused by multi resistant E. faecium, however, did not only increase the total burden of nosocomial enterococcal infections, butalso resulted in a partial replacement of E. faecalis by E. faeciumas a cause of hospital-associated infections. Several studies showed that an increased proportion of nosocomial enterococcal infections caused by E. faecium.

\section{CONCLUSIONS AND RECOMMENDATIONS}

$23 \%$ of the hospitalized pediatrics patients carried enterococci in their gastrointestinal tracts and E. faecium was the predominant species.E. faecium showed highest resistance rate to vancomycin. Therefore, regular monitoring for the presence of VRE in both hospitals and the community, effective strategies for the prevention of antimicrobial resistance should be practiced. Unnecessary use of antibiotics and ignorance infection control measure should be stopped.

\section{FUNDING}

This work was funded from Jimma University core budget.

\section{AUTHORS' CONTRIBUTIONS}

SG-performed the laboratory activities. BF, SG and TA- analyzed the data. TA-wrote the manuscript. All authors read and approved the final manuscript.

\section{ACKNOWLEDGMENT}

We would like to thank Debre Berhan University for giving this opportunity and availing the resources. We also thank staffs and head of Debre Berhan university specialized hospital for their help during data collection process.

\section{REFERENCES}

[1] Abdul hakim Abamecha, Beyene Wondafrash and Alemseged Abdissa Antimicrobial resistance profile of Enterococcus species isolated from intestinal tracts of hospitalized patients in Jimma, Ethiopia Abamecha et al. BMC Res Notes (2015) 8:213

[2] Boost M, Lai L, O'Donoghue M (2004) Drug resistance in fecal enterococci in Hong Kong. J Infect Chemother 10:326-330

[3] Arias CA, Murray BE (2008) Emergence and management of drug-resistant Enterococcal infections. Expert Rev Anti Infect Ther 6:637-655.

[4] Sood S, Das MM, Kapil A (2008) Enterococcal infections and antimicrobial resistance. Indian J Med Res 128:111-121

[5] Shah L, Mulla S, Patel KG, Rewadiwala S (2012) Prevalence of enterococci with higher resistance level in a tertiary care hospital: a matter of concern. Natl J Med Res 2:25-27

[6] Manero A, Blanch AR (1999) Identification of Enterococcus species with a biochemical key. Appl Environ Microbiol 65:4425-4430

[7] Clinical and Laboratory Standards Institute (2007) Performance standards for antimicrobial susceptibility testing, informational supplement (M100-S17), 17th edn. Clinical and Laboratory Standards, Wayne

[8] Silverman J, Thal LA, Perri MB, Bostic G, Zervos MJ (1996) Epidemiologic evaluation of antimicrobial resistance in community-acquired Enterococci. J ClinMicrobiol 36:830-882

[9] Cai Y, Chan PJ, Fisher D, Hsu L, Koh T, Krishnan P (2012) Vancomycin-resistant Enterococci in Singaporean hospitals: 5-year results of a multi-centre surveillance programme. Ann Acad Med Singapore 41:77-81

[10] Kapoor L, Randhawa VS, Deb M (2005) Antimicrobial resistance of enterococcal blood isolates at a pediatric care hospital in India. Jpn J Infect Dis 58:101-103 
[11] Udo E, Al-Sweih N, John P, Chugh TD (2002) Antibiotic resistance of enterococci isolated at a teaching hospital in Kuwait. DiagMicrobiol Infect Disn 43:233-238

[12] Boost M, Lai L, O’Donoghue M (2004) Drug resistance in fecal enterococci in Hong Kong. J Infect Chemother 10:326-330

[13] Eduardo E, Freitas A, Reiter K, Luts L, Afonso Barth A (2009) Identification, antimicrobial reistance and genotype characterization of Enterococcus spp. in Porto Alegre, Brazil. Braz J Microbiol 40:693-700

[14] Dr. Abdelraouf A. Elmanama,Dr. Adnan Al-Hindi. Vancomycin-Resistant Enterococci in Fecal Samples from Hospitalized Patients and Non- Hospitalized Individuals in Gaza City

[15] Dan M, Poch F, Leibson L, Smetana S, Priel I (1999) Rectal colonization with vancomycin-resistant enterococci among high-risk patients in an Israeli hospital. J Hosp Infect 43:231-238

[16] Sood S, Das MM, Kapil A (2008) Enterococcal infections and antimicrobial resistance. Indian J Med Res 128:111-121

[17] Kholy AE, Baseem H, Hall GS, Procop GW, Longworth DL (2002) Antimicrobial resistance in Cairo, Egypt 1999-2000: a survey of five hospitals. J AntimicrobChemother 51:625-630

[18] Assadian O, Askarian M, Stadler M, Shaghaghian S (2007) Prevalence of vancomycin-resistant enterococci colonization and its risk factors in chronic hemodialysis patients in Shiraz, Iran. BMC Infect Dis 7:52-56

[19] Von Gottberg A, van Nierop W, Duse A, Kassel M, McCarthy K, Brink A (2000) Epidemiology of glycopeptide-resistant enterococci colonizing high-risk patients in hospitals in Johannesburg, Republic of South Africa. J ClinMicrobiol 38:905-909

[20] Yang J, Lee D, Kim Y, Kang B, Kim K, Ha N (2007) Occurrence of the van genes in Enterococcus faecalisand Enterococcus faeciumfrom clinical isolates in Korea. Arch Pharm Res. 30:329-336

[21] Coleri A, Cokmus C, Ozcan B, Akcelik M, Tukel C (2004) Determination of antibiotic resistance and resistance plasmids of clinical Enterococcus species. J Gen ApplMicrobiol 50:213-2

Citation: T. Asfaw, "Magnitude of Drug-Resistant Enterococcus Species from ntestinal Tracts of Hospitalized Pediatric Patients in Debreberhan Referral Hospital, Debreberhan, Ethiopia", International Journal of Research Studies in Microbiology and Biotechnology (IJRSMB), vol. 5, no. 1, pp. 1-6, 2019. Available: DOI: http://dx.doi.org/10.20431/2454-9428.0501001.

Copyright: (C) 2019 Authors. This is an open-access article distributed under the terms of the Creative Commons Attribution License, which permits unrestricted use, distribution, and reproduction in any medium, provided the original author and source are credited. 